\title{
Anti-inflammatory efficacy of Berberine Nanomicelle for improvement of cerebral ischemia: formulation, characterization and evaluation in bilateral common carotid artery occlusion rat model
}

Roza Azadi', Seyyedeh Elaheh Mousavi ${ }^{2 *}$, Negar Motakef Kazemi ${ }^{1}$, Hasan Yousefi-Manesh², Seyed Mahdi Rezayat ${ }^{2,3}$ and Mahmoud Reza Jaafari ${ }^{4}$

\begin{abstract}
Background: Berberine (BBR) is a plant alkaloid that possesses anti-inflammatory and anti-oxidant effects with low oral bioavailability. In this study, micelle formulation of BBR was investigated to improve therapeutic efficacy and examined its effect on the secretion of inflammatory cytokines in cerebral ischemia in the animal model.

Material and methods: Nano formulation was prepared by thin-film hydration method, and characterized by particle size, zeta potential, morphology, encapsulation efficacy, and drug release in Simulated Gastric Fluid (SGF) and Simulated Intestine Fluid (SIF). Then, Wistar rats were pretreated with the drug $(100 \mathrm{mg} / \mathrm{kg})$ and nano-drug (25, $50,75,100 \mathrm{mg} / \mathrm{kg}$ ) for 14 days. Then, on the fourteenth day, stroke induction was accomplished by Bilateral Common Carotid Artery Occlusion (BCCAO); after that, Tumor Necrosis Factor - Alpha (TNF-a), Interleukin - 1 Beta $(\mathrm{IL}-1 \mathrm{~B})$, and Malondialdehyde (MDA) levels were measured in the supernatant of the whole brain, then the antiinflammatory effect of BBR formulations was examined.

Result and discussion: Micelles were successfully formed with appropriate characteristics and smaller sizes than $20 \mathrm{~nm}$. The Poly Dispersity Index (PDI), zeta potential, encapsulation efficacy of micelles was $0.227,-22 \mathrm{mV}, 81 \%$, respectively. Also, the stability of nano micelles was higher in SGF as compared to SIF. Our outcomes of TNF-a, IL1B, and MDA evaluation show a significant ameliorating effect of the Berberine (BBR) and BBR-loaded micelles in pretreated groups.
\end{abstract}

\footnotetext{
* Correspondence: semousavi@sina.tums.ac.ir

${ }^{2}$ Department of Pharmacology, School of Medicine, Tehran University of Medical Sciences, Tehran, Iran

Full list of author information is available at the end of the article
}

C C The Author(s). 2021 Open Access This article is licensed under a Creative Commons Attribution 4.0 International License, which permits use, sharing, adaptation, distribution and reproduction in any medium or format, as long as you give appropriate credit to the original author(s) and the source, provide a link to the Creative Commons licence, and indicate if changes were made. The images or other third party material in this article are included in the article's Creative Commons. licence, unless indicated otherwise in a credit line to the material. If material is not included in the article's Creative Commons licence and your intended use is not permitted by statutory regulation or exceeds the permitted use, you will need to obtain permission directly from the copyright holder. To view a copy of this licence, visit http://creativecommons.org/licenses/by/4.0/ The Creative Commons Public Domain Dedication waiver (http://creativecommons.org/publicdomain/zero/1.0/) applies to the data made available in this article, unless otherwise stated in a credit line to the data. 


\begin{abstract}
Conclusion: Our experimental data show that pretreated groups in different doses (nano BBR 100, 75, 50 mg/kg, and BBR $100 \mathrm{mg} / \mathrm{kg}$ ) successfully showed decreased levels of the inflammatory factors in cerebral ischemia compared with the stroke group and pretreated group with nano BBR in the dose of $25 \mathrm{mg} / \mathrm{kg}$. Nano BBR formulation with a lower dose can be a better candidate than conventional BBR formulation to reduce oxidative and inflammatory factors in cerebral ischemia. Therefore, BBR-loaded micelle formulation could be a promising protective agent on cerebral ischemia.
\end{abstract}

Keywords: Berberine, BCCAO model, Cerebral ischemia, Nano formulation, Anti-inflammation

\section{Introduction}

Many studies have been done on cerebral ischemia and all of them have confirmed its morbidity and mortality worldwide [1]. Cerebral ischemia occurs due to a blockage in the carotid arteries, which results in damage to brain tissue. Results indicate factors such as oxygen-free radicals and inflammatory cytokines (such as TNF- $\alpha$, IL$1 ß)$ and Malondialdehyde (MDA) as a marker of oxidative stress that plays an important role in damaging brain tissue and neurons due to cerebral ischemia [2-4]. There is evidence that TNF-a and IL-1B levels in the brain increase many-fold (up to 40 or 60 times) during the first $24 \mathrm{~h}$ after inducing stroke [5]. Therefore, it is necessary to recognize safe compounds with high and effective therapeutic efficacy in reducing inflammation during the stroke.

Berberine (BBR), an isoquinoline alkaloid extracted from Coptidis rhizome and Cortex Phellodendron, is one of the herbal and safe compounds $\left(\mathrm{C}_{20} \mathrm{H}_{18} \mathrm{NO}_{4}{ }^{+}\right)$ which exhibits anti-oxidative and anti-inflammatory effects on brain diseases such as ischemia [6-9], Alzheimer [10], and tumors [11]. Also, it has been reported that BBR possesses effects of antibacterial [12], Antioxidant [13], antiviral [14], and anticancer [15]. However, the therapeutics effectiveness of BBR is restricted due to its hydrophobic nature and poor water solubility [16]. Pharmacokinetic studies of BBR in animals indicate that BBR has low plasma concentration for $48 \mathrm{~h}$ because of its poor absorption rate in the gut wall about $33.6 \%$ within $1 \mathrm{~h}$ [17]. Also, it is metabolized rapidly in the liver that is the main metabolic site in the body, and after BBR absorption, the clearance of BBR from blood is fast. In the rat's body, it is quickly transferred to the liver and bile through active transportation, then rapidly will be biotransformed $[18,19]$. BBR has metabolized in the rat liver via phase I demethylation and phase II glucuronidation, then apparently excreted through the duodenum in bile [17]. Current findings indicate that the toxicity of BBR is mainly associated with intravenous administration.

Also, according to results in the drug bank, the BBR melting point is $145^{\circ} \mathrm{C}$, its water solubility is 0.000354 $\mathrm{mg} / \mathrm{mL}, \log \mathrm{P}$ is -1.3 , and bioavailability is 1 [20]. Therefore, to overcome these challenges, we need a novel formulation in the drug delivery system to improve drug solubility and efficiency.

As mentioned above, mucus on the gut wall is a barrier to the absorption of hydrophobic particles. So, applying nanotechnology and nanocarriers can be an effective way to overcome this challenge. Various studies were accomplished on nanocarriers for BBR delivery with an approach to the improvement and treatment of different types of disease. Some inorganic and organic nanocarriers used for BBR including gold nanoparticles, magnetic mesoporous nanoparticles [21], polymers [22], lipid-based [23], and so forth. Also, plentiful studies were done on the therapeutic effects of BBR on stroke ischemia, but none of them used nano-formulation. For instance, increased bioavailable BBR attenuated cerebral ischemia/reperfusion (I/R) injury in rats [24]. Research by Pishva and et al. showed the effect of berberine nano micelle on inflammatory mediators for the improvement of cirrhotic cardiomyopathy in rats. It has been recognized that nano micelle berberine compared with berberine possesses a better anti-inflammatory potential in cirrhotic injuries [25].

Lately, the hydrophobic drugs encapsulating micelles as nanocarriers have attracted much attention for drug delivery applications [26]. Micelles are amphiphilic colloidal structures with particle diameters from 5 to 100 $\mathrm{nm}$ range. The core of the micelle is formed by the hydrophobic fragments of amphiphilic molecules, whereas the micelle's shell consists of hydrophilic micellar molecule fragments [27]. Micelles possess advantages for oral drug delivery, such as the ability to solubilize hydrophobic drugs due to their unique structure (coreshell) resulting in enhancing drug effectiveness without change or disruption in drug formulation [28-31]. So, the design of the drug delivery system based on micelles could show promising performances in oral administration by providing a high level of therapy.

The present study aimed to develop the drug formulation with an approach to nanoencapsulation and evaluation of its effect on the secretion of inflammatory mediators in stroke ischemia in the rat.

We tried focusing on the preparation of nano formulation by thin-film hydration method and its characterization, then after inducing stroke, levels of 
inflammation and oxidative factors (TNF- $\alpha$, IL- $1 \beta$, MDA) were measured in brain tissue. Thus, the aim of this study was to preparation and comparison of BBR and BBR-loaded micelle formulations and survey their anti-inflammatory effects on cerebral ischemia-induced with Bilateral Common Carotid Artery Occlusion (BCCAO) model in the rats.

\section{Material and methods}

Deoxycholate and BBR (98\% purity) were purchased from Sigma. Ketamine hydrochloride and xylazine were from Alfasan (Holland). The chemical used including $\mathrm{NaOH}, \mathrm{KH}_{2} \mathrm{PO}_{4}, \mathrm{NaCl}, \mathrm{HCl}$, and other materials and solvents were analytical grades. Equipment was used including rotary (Buchi, Switzerland), centrifuge, filter amicon $3000 \mathrm{KD}$, and spectrophotometer UV-Vis (UV160 A, SHIMADZU - Japan).

\section{Preparation of BBR-loaded micelle solution}

The Thin-film hydration method was used for the preparation of BBR-loaded micelle solution [32]. Briefly, in a round-bottom flask BBR $(1 \% \mathrm{w} / \mathrm{w})$ and deoxycholate $(49 \% \mathrm{w} / \mathrm{w})$ as the surfactant, was mixed with a minimum amount of methanol as an organic solvent. The rotary evaporator was applied for more than $2 \mathrm{~h}$ to evaporate solvent under reduced pressure and to deposit thin film. Then, in the hydration phase, deionized water $(50 \% \mathrm{w} /$ w) was added to it, and bain-marie was applied to $50{ }^{\circ} \mathrm{C}$ while stirring was done for $20 \mathrm{~min}$ at the same temperature until a clear solution was obtained that was light yellow.

\section{Characterization}

Dynamic light scattering reported size and size distribution, PDI, Zeta potential of nano micelles (Malvern Instruments, UK). The transmission electron microscope (TEM) was applied to show the size of nano micelle formulations (PHILIPS CM 300, Netherlands).

In transmission electron microscope (TEM), samples were diluted in ethanol and placed under dispersion for $20 \mathrm{~min}$. Then, nano micelles (1 drop) are deposited onto a carbon-coated copper grid. After dried, the samples viewed and photographed with a Phillips cm300 electron microscope. For the determination of encapsulation efficiency, an indirect method was used. Briefly, after centrifugation of micelles at $4000 \times \mathrm{g}$ for $30 \mathrm{~min}$, the concentration of BBR in the samples was determined by spectroscopy UV-Vis in $\lambda \max =348$ nm (UV-160 A, SHIMADZU - Japan). The drug encapsulation efficacy was calculated by the following equations [33]:

$$
E E \%=\frac{\text { drug concentration post-filterate drug }}{\text { initial concentration of drug }} \times 100
$$

\section{Drug release study}

The Release pattern of BBR from nano micelles was investigated in SGF and SIF. To prepare the SGF, $246 \mu \mathrm{L}$ $\mathrm{HCl}$ and $200 \mathrm{mg} \mathrm{NaCl}$ were added to $60 \mathrm{ml}$ deionized water (DW), and $\mathrm{pH}$ was adjusted at 2 , then the final volume was filled to $100 \mathrm{~mL}$ with DW. Also, SIF was prepared by dissolving $680 \mathrm{mg}$ of $\mathrm{KH}_{2} \mathrm{PO}_{4}$ and $61.6 \mathrm{mg}$ of $\mathrm{NaOH}$ in $60 \mathrm{~mL} \mathrm{DW}$, and $\mathrm{pH}$ was adjusted at 6.5 . Then the final volume was filled to $100 \mathrm{~mL}$ with DW. Samples were diluted in a ratio of 1:10 in SIF and SGF and incubated at $37^{\circ} \mathrm{C}$. Then sampling was carried out at time points $0,30 \mathrm{~min}, 1,2,4,8,24 \mathrm{~h}$ [34]. Then the determination of BBR was accomplished by spectroscopy UV-Vis in $\lambda \max =348 \mathrm{~nm}$ (UV-160 A, SHIMADZU Japan).

\section{Animal groups and ethical considerations}

In this project, 96 adult male Wistar-rats weighing 200$220 \mathrm{~g}$ (aged 8-10 weeks) were purchased from the Pharmacology School of Tehran University of Medical Sciences. All of them were kept in an animal house under standard laboratory conditions at the temperature of $22 \pm 1{ }^{\circ} \mathrm{C}$ and humidity of $80 \%$, with a typical $12 \mathrm{~h}$ light/dark cycle, and allowed to have free access to food and water. All phases of the experiment were approved by the ethics committee of Tehran University of Medical Sciences for the maintenance and application of laboratory animals (IR.TUMS.MEDICINE.REC.1398.593). We do all attempts to minimize animal suffering, and during the experiments to collect brain tissue samples, the animals were euthanized completely by carbon dioxide gas. Then, animal carcasses were disposed of with hospital waste.

The rats were randomly divided into 8 groups (12 rats in each group) as follow:

(I) Control group

(II) Stroke group

(III) Nano micelle group (without drug)

(IV) Pretreated group with BBR $(100 \mathrm{mg} / \mathrm{kg})$

(V) Pretreated group with nano BBR $(100 \mathrm{mg} / \mathrm{kg})$

(VI) Pretreated group with nano BBR $(75 \mathrm{mg} / \mathrm{kg})$

(VII) Pretreated group with nano BBR $(50 \mathrm{mg} / \mathrm{kg})$

(VIII)Pretreated group with nano BBR $(25 \mathrm{mg} / \mathrm{kg})$

The control group hasn't received any drug and no induction has been made on it. The stroke group induction has been performed but has not received the drug. Also, groups 4-8 were treated orally with BBR and nano-BBR for 14 days with concentrations of $25,50,75$, 
$100 \mathrm{mg} / \mathrm{kg}$. For oral administration of berberine hydrochloride, it (approximately $1 \%$ of body weight) was dissolved in water [35]. In this study, the vehicle was micelle and the solvent that Berberine and Nano Berberine were diluted in that was water.

\section{Stroke inducing by BCCAO (bilateral common carotid artery occlusion) model}

For the inducing of cerebral ischemia (CI), rats were anesthetized by an intraperitoneal (i.p) injection of 50 $\mathrm{mg} / \mathrm{kg}$ ketamine and $2-8 \mathrm{mg} / \mathrm{kg}$ xylazine. The animals were placed on their back, and their tail and paws were fixed using adhesive tape. A vertical incision ( $1 \mathrm{~cm}$ length) was performed in the neck of each rat, then the bilateral common carotid arteries were revealed, and both carotid arteries were carefully separated from the vagal nerve. Also, it is crucial to avoid any manipulations of the vagal nerve. A 5-0 silk suture loop was made around each carotid artery. Both carotid arteries were occluded for $30 \mathrm{~min}$. Then sixty (60) minutes reperfusion period was initiated. During the reperfusion period, the wounds were sutured and with an anti-septic solution were disinfected, and kept in a separate cage. By finishing the reperfusion period, the rats were decapitated and the whole brain removed for molecular analysis [36-38].

\section{Biochemical assays}

Rats were decapitated under deep anesthesia, the whole brains were removed, and all tissues were stored at $80^{\circ} \mathrm{C}$ for later biochemical assays. The animal's whole brains were homogenized in buffer TRIS hydrochloride (TRIS HCL), Safety Data Sheet (SDS), Dithiothreitol (DTT), nonyl phenoxy polyethoxyl ethanol (NP40), and glycerol, then centrifuged for $5 \mathrm{~min}$ at the room temperature 20 to $25^{\circ} \mathrm{C}\left(68\right.$ to $\left.77^{\circ} \mathrm{F}\right)$ [39], after that, the levels of IL- $1 \beta$ and TNF- $\alpha$ in the supernatant were detected with ELISA kits, also the activity of MDA (as a marker for lipid peroxidation) in the supernatant was measured using the commercial kit according to the manufacturer's instructions.

\section{Assay kits were purchased from international and domestic commercial companies (ZellBio GmbH, Ulm, Germany) \\ Statistical analysis}

Data analysis was carried out using GraphPad Prism version 7 software. All data were analyzed using one-way analysis of variance (ANOVA) and Tukey post-hoc. Data were presented as Mean \pm SEM. Statistically, differences with $p<0.05$ were recognized as significant.

\section{Results}

\section{Characterization}

BBR-loaded micelle formulation successfully was prepared. The dynamic light scattering was used to find out the three of the main parameters of particle size (based on intensity, Number, and Volume), PDI, and zeta potential of nano micelles (Figs. 1, 2, 3 and 4). DLS results showed an average size about of $12 \mathrm{~nm}$, zeta potential (which represents surface charge) and PDI (which represents the size distribution and system uniformity) were $-22 \mathrm{mv}$ and 0.227 , respectively. Also, the size less than $20 \mathrm{~nm}$ was confirmed by TEM (Fig. 5). The samples were photographed with a Philips cm300 electron microscope (Philips cm300/ $200 \mathrm{k} /$ Holland). Diluted nano micelles were dispersed for $20 \mathrm{~min}$ and deposited onto a carbon-coated copper grid. After drying, the images were taken, and the morphology of nano micelles using TEM revealed that they have spherical-like shapes.

Encapsulation of BBR into nano micelles was measured by using the centrifugation method, and parameters were calculated according to eq. 1. EE\% was calculated as $81 \%$, which suitable for the objective of the current study.

\section{Drug release study}

Percent of drug released from nano micelle formulation in SGF and SIF solutions. Drug release studies in SGF and SIF were evaluated within 24-h sampling, but the maximum drug residence time in the stomach is $2 \mathrm{~h}$, so the results of the first $2 \mathrm{~h}$ are important for the survey of drug behavior in SGF.

The level of BBR didn't fluctuate much in the first $4 \mathrm{~h}$ in SGF. However, it was decreased to $71 \%$ after 8 $h$ of incubation. The stability of nano micelles was higher in SIF as compared to SGF. In SIF, the concentration of BBR decreased from $97 \%$ at $0 \mathrm{~h}$ to $86 \%$ after $8 \mathrm{~h}$. As the results showed, this time is well enough for nano micelles to pass the stomach and reach the intestine, where they can absorb with different mechanisms.

\section{Effects of nano BBR-loaded micelle on TNF- $a$, IL-1ß, and MDA}

To evaluate the anti-inflammatory effect of berberine, we examined BBR and nano BBR on cytokines and the marker of stress oxidative secreted into brain tissue within cerebral ischemia. Results were shown in (Figs. 6, 7 and 8). After treatment with various concentrations of BBR $(100 \mathrm{mg} / \mathrm{kg})$ and nano BBR (25, $50,75,100 \mathrm{mg} / \mathrm{kg}$ ) for 14 days, brain tissues were analyzed by ELISA kits to examine whether the treatments affect the release of inflammatory cytokines. According to the results, the level of TNF- $\alpha$ (from 9 $\mathrm{pg} / \mathrm{mg}$ in nano micelle group $(* * *)$ to $6 \mathrm{pg} / \mathrm{mg}$ in the 


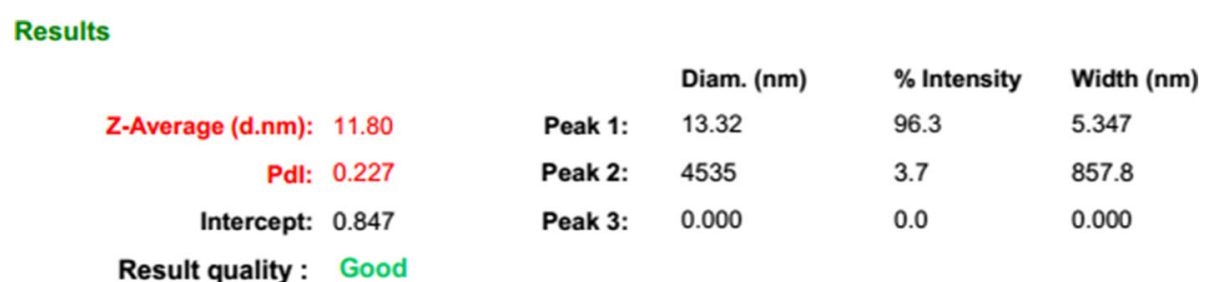

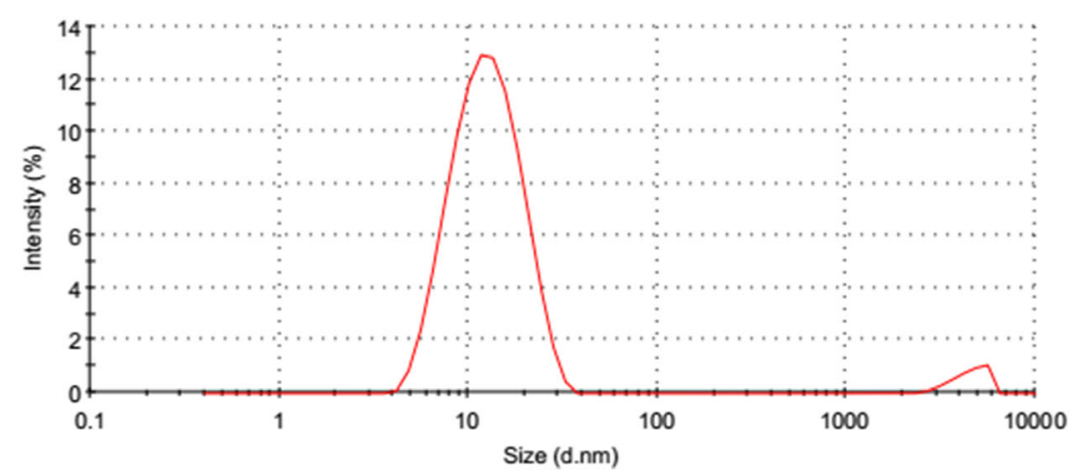

Fig. 1 Size distribution diagram based on Intensity

treated group (\#\#) with nano BBR concentration of $100 \mathrm{mg} / \mathrm{kg}$ ), IL_1ß (from $6 \mathrm{pg} / \mathrm{mg}$ in nano micelle group to $3.8 \mathrm{pg} / \mathrm{mg}$ in the treated group (\#\#\#) with nano BBR concentration of $100 \mathrm{mg} / \mathrm{kg}$ ), and MDA (from $4.8 \mathrm{pg} / \mathrm{mg}$ in nanomicelle group to $3.6 \mathrm{pg} / \mathrm{mg}$ in treated groups with nano BBR concentration (\#\#\#) of 100 and $75 \mathrm{mg} / \mathrm{kg}$ ) were reduced in a dose-dependent manner in, indicating the inhibited effect by BBR on the secretion of inflammatory cytokines.

Also, our results show that not only there were no significant differences between the stroke group $(* * *)$ and nano micelle group (without drug) in every three parameters, but also significant difference was observed between the stroke group $(* *)$ and pretreatment groups (@, @@).

\section{Results}

$\begin{array}{rlllll} & & & \text { Diam. }(\mathrm{nm}) & \% \text { Number } & \text { Width }(\mathrm{nm}) \\ \text { Z-Average (d.nm): } & 11.80 & \text { Peak 1: } & 6.416 & 100.0 & 1.946 \\ \text { Pdl: } & 0.227 & \text { Peak 2: } & 0.000 & 0.0 & 0.000 \\ \text { Intercept: } & 0.847 & \text { Peak 3: } & 0.000 & 0.0 & 0.000\end{array}$

Result quality : Good

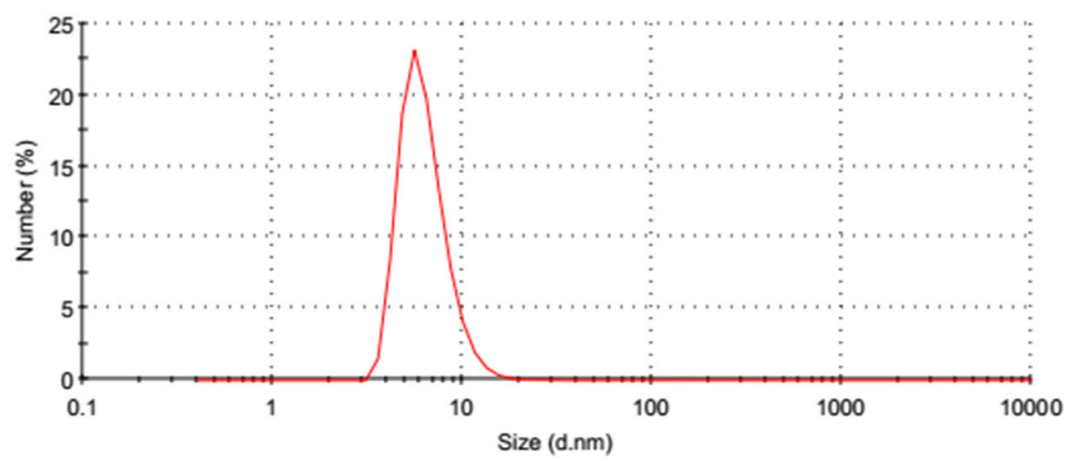

Fig. 2 Size distribution diagram based on Number 


\begin{tabular}{rlllll}
\multicolumn{1}{l}{ Results } & & & & & \\
Z-Average (d.nm): & 11.80 & & Diam. (nm) & $\%$ Volume & Width (nm) \\
Pdl: & 0.227 & Peak 1: & 8.575 & 99.8 & 3.430 \\
Intercept: & 0.847 & Peak 3: & 0.000 & 0.2 & 949.6 \\
& & & & 0.0 & 0.000
\end{tabular}

Result quality : Good

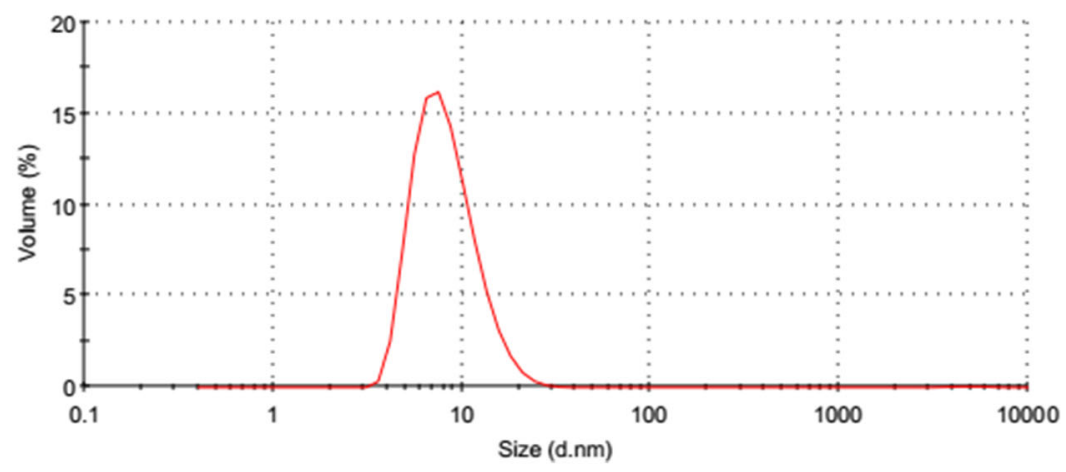

Fig. 3 Size distribution diagram based on Volume

It has been accepted that berberine can decrease inflammatory agents-induced IL-1B and TNF-a ensuing inflammation in cerebral ischemia [40]. In the present work, studies have shown that Induction of stroke caused a significant increase in TNF-a, IL-1B, and MDA levels compared to the control group $(p<0.001)$. Also observed increase of cytokines and the marker of oxidative stress in the treated group (nano BBR $25 \mathrm{mg} / \mathrm{kg}$ ) (\$\$, \&\&\&) and nano micelle (without drug) group compared to the control group $(\mathrm{p}<0.001)$.

On the other hand, pretreated groups (@, @@) (BBR $100 \mathrm{mg} / \mathrm{kg}$, nano BBR $100 \mathrm{mg} / \mathrm{kg}(p<0.01), 75 \mathrm{mg} / \mathrm{kg}$,

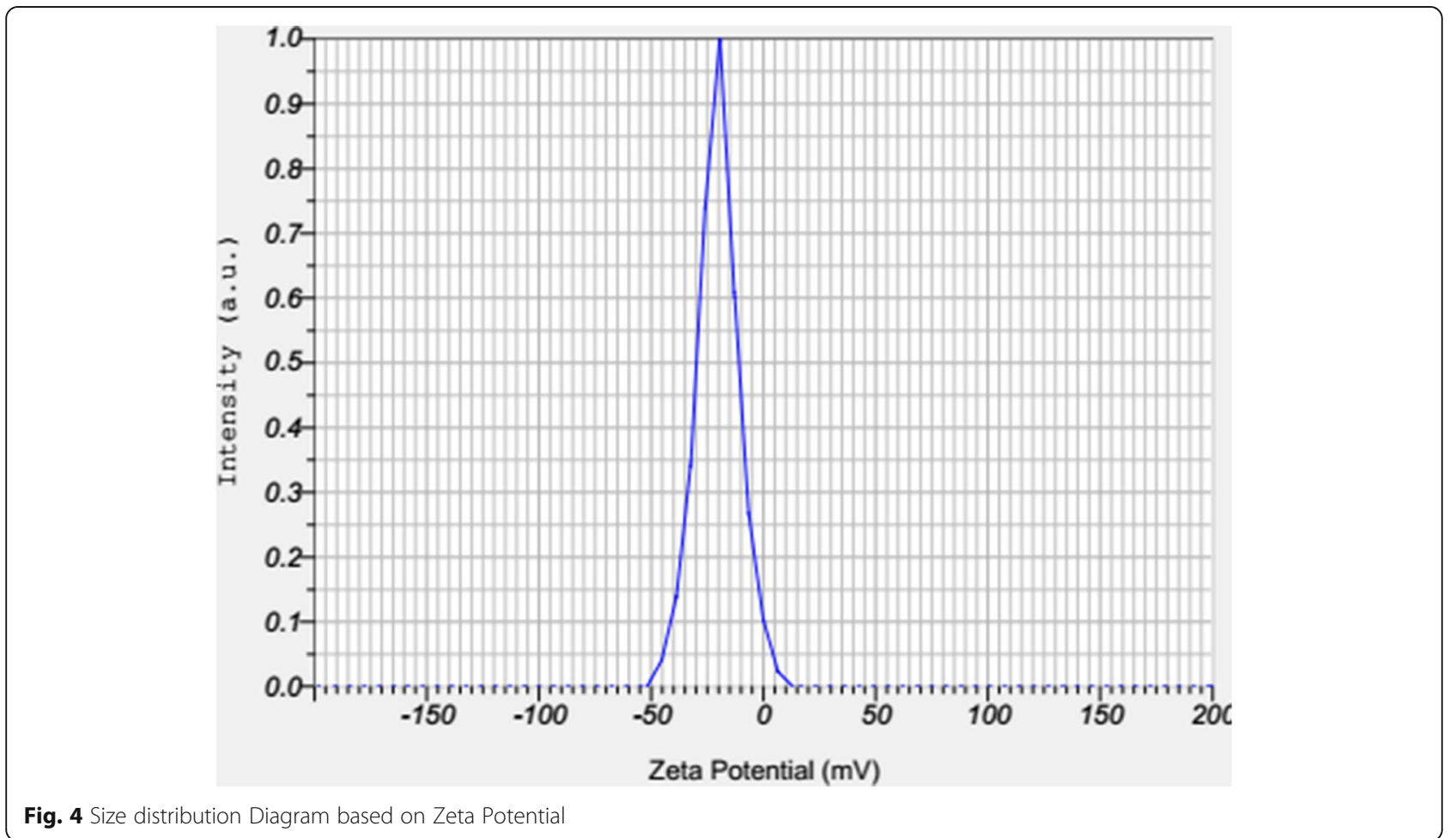




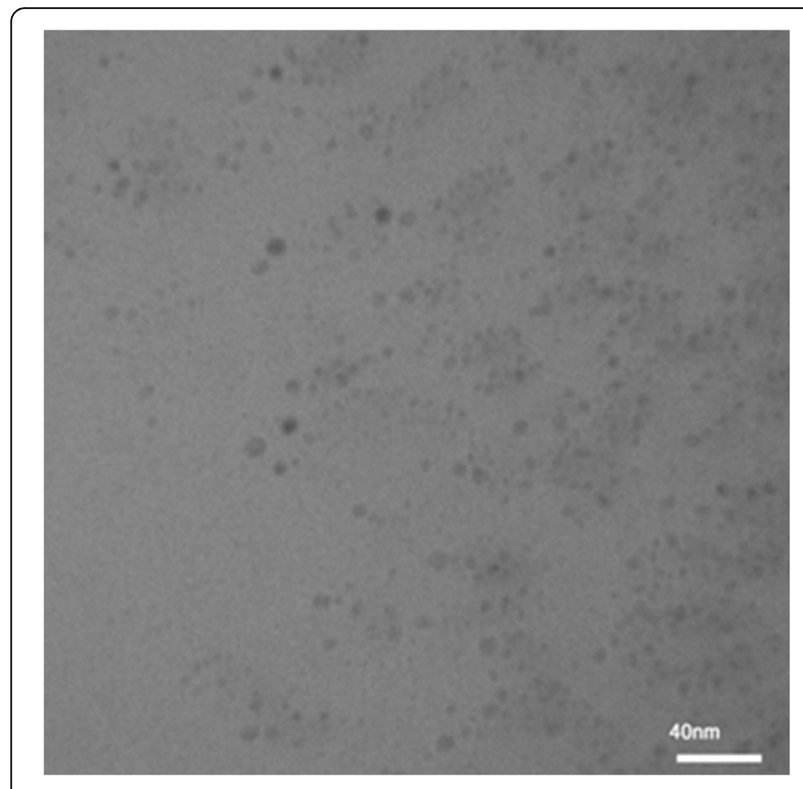

Fig. 5 TEM image of BBR Loaded micelle

and $50 \mathrm{mg} / \mathrm{kg}(p<0.05))$ showed a significant reduction in TNF- $\alpha, \mathrm{IL}-1 \mathrm{~B}$, and MDA levels compared to the stroke group.

\section{Discussion}

Different studies reported that several mechanisms have to alleviate cerebral ischemic injury by berberine through its inhibition effects on the level of inflammatory cytokines and oxidative stress including TNF-a, IL-1B, and MDA. Also, several studies have reported similar findings to this one as well, such as Nasseri Maleki et al. reported Berberine might be used as a potential therapeutic agent to reduce inflammation [40]. In addition, Singh and Chopra have shown antiinflammatory effects of berberine against cerebral ischemia in animal models [41].

The induction of the BCCAO model leads to commence the inflammation pathways by occlusion the blood flow to the brain. In addition, the reperfusion time after an interruption in blood circulation to the brain is a key step to produce the reactive oxygen species (ROS) in the brain cells in particular. Therefore, measurement of TNF-a and IL-1B for assessment of the antiinflammatory effects, and MDA to assay the effect of drugs in stress oxidative condition in general and on reactive oxygen species (ROS), in particular, could be a usable way [42].

The aim of this study was to emphasize 2 main parameters including preparation and survey of antiinflammatory effect BBR-loaded micelle nano formulation on cerebral ischemia.

First, BBR-loaded micelles successfully were prepared by the thin-film hydration method. The size of prepared micelles was approximately $12 \mathrm{~nm}$ and shows a good distribution. Simoes et al. reported this small size of micelles provides enhanced water solubility of hydrophobic drugs, increased absorption of free drugs, and they

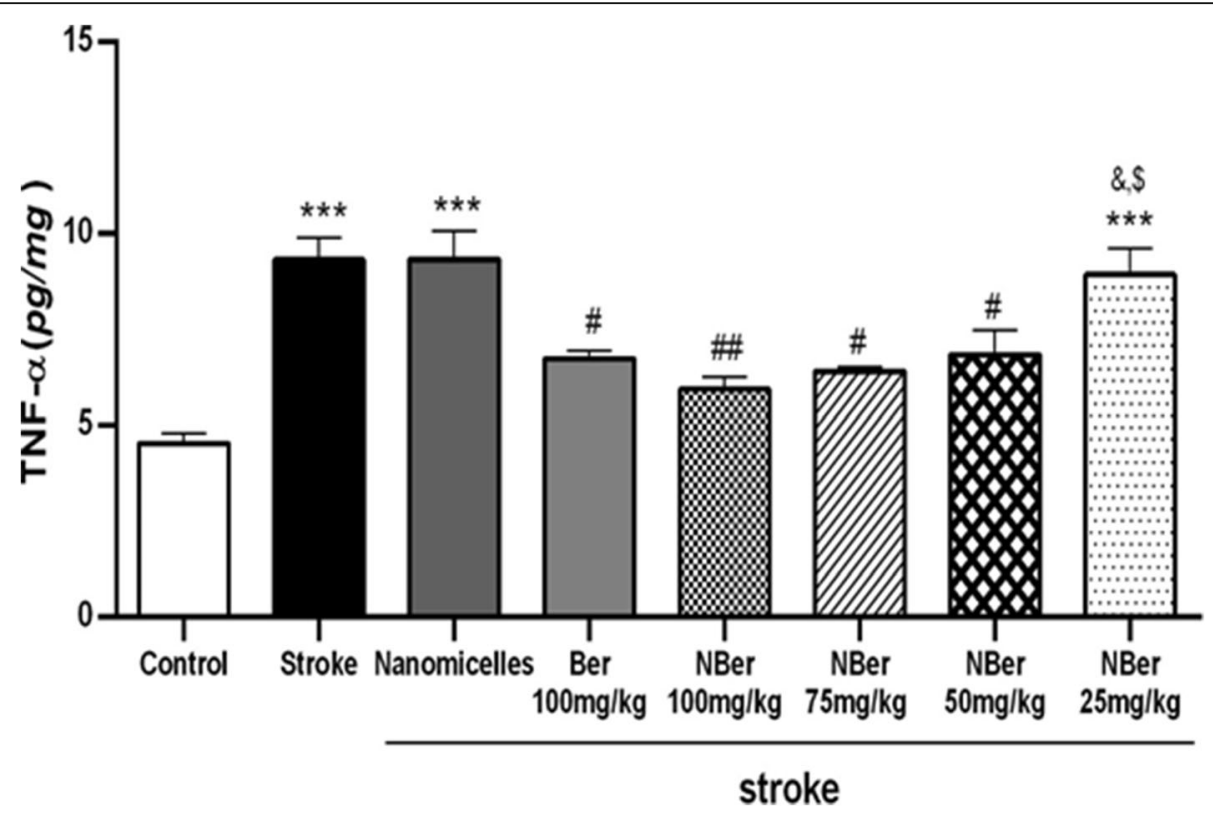

Fig. 6 Rate changes of TNF-a in different groups after inducing stroke in rat. Rats were pretreated with BBR (100 mg/kg, oral administration) and BBR-loaded micelle $(100,75,50,25 \mathrm{mg} / \mathrm{kg})$ for 14 days before ischemia. ELISA kit was used to detect the level of TNF-a after ischemia. Results show concentrations of BBR $100 \mathrm{mg} / \mathrm{kg}$, and nano BBR $100 \mathrm{mg} / \mathrm{kg}(P<0.01), 75$ and $50 \mathrm{mg} / \mathrm{kg}(P<0.05)$ had more reduction in TNF-a level compared to the stroke group 


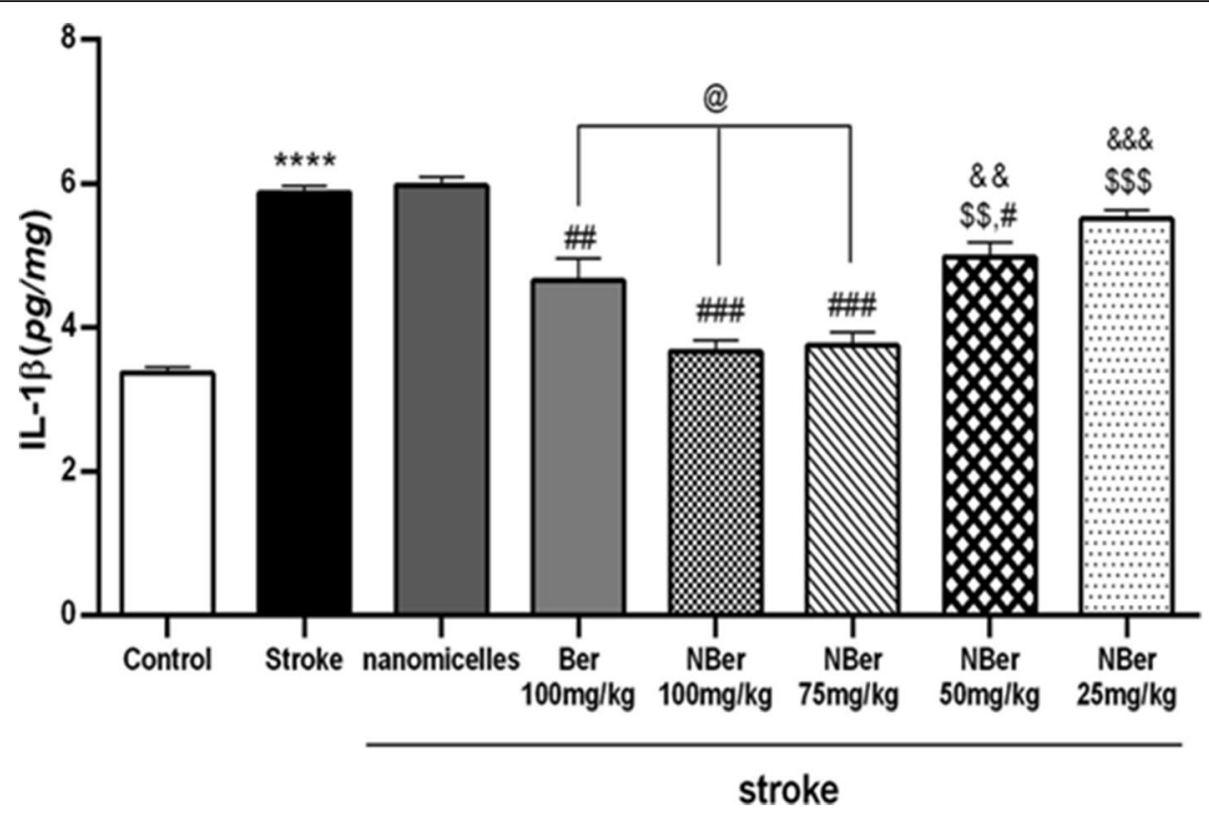

Fig. 7 Rate changes of IL-1B in different groups after inducing stroke in rat. $N=8$ for each group. $P<0.01, P<0.05$ compared to stroke group. Data are presented increase the level of IL-1B in treated group (nano BBR $25 \mathrm{mg} / \mathrm{kg}$ ) and nano micelle (without drug) group compared to the control group $(p<0.001)$

release the drug in the target site of the gastrointestinal tract together with a high concentration gradient nearby the epithelium [43]. For these reasons, the small size of micelles has particular importance in oral administration in the drug delivery system. Also, zeta potential shows that the prepared formulation has a negative surface charge that makes it stable against aggregation.

The results presented in this study demonstrated that $81 \%$ of BBR molecules successfully entrapped within the core of the micelle. To calculate it, we examined the centrifuge method for the separation of entrapped BBR from the micellar system. Also, we confirmed that the centrifuge method compared to other methods (gel chromatography and dialysis method) is the most appropriate for the separation of free drugs from the micellar system [44]. Drug release studies have shown that BBR was released in a declining trend from nano micelle formulations in SGF and SIF, and they are stable for at least

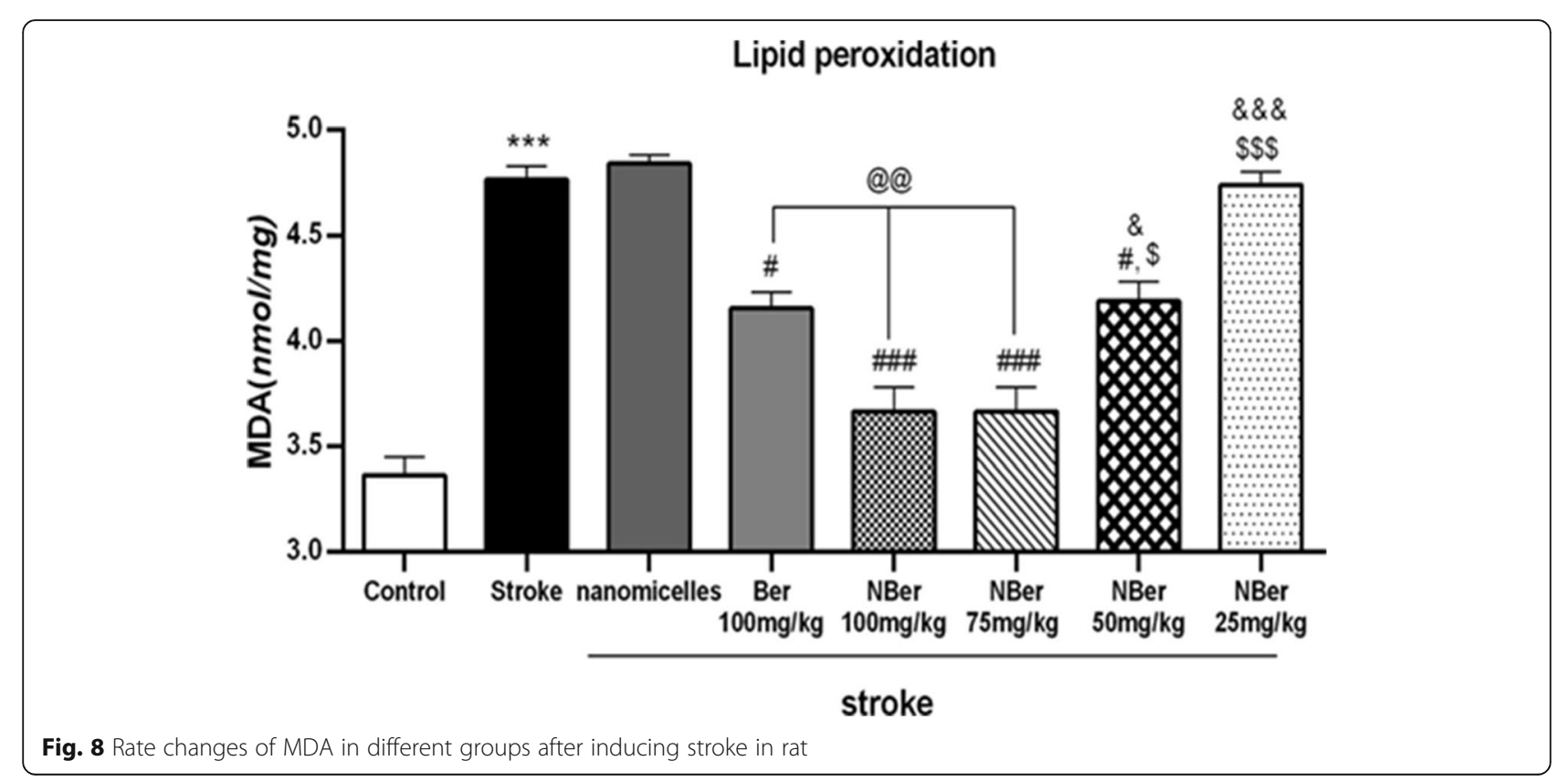


$4 \mathrm{~h}$. Moreover, the BBR release rate from nano micelle formulation was higher in SIF than in SGF.

Second, we compared the effect of BBR and BBRloaded micelle on TNF-a, IL-1B (inflammations factors), and MDA (marker of oxidative stress) due to inducing cerebral ischemia in the rat. BCCAO model was used to induce stroke into rat's brain. An increase in inflammation cytokines and MDA have been reported after inducing stroke [45]. In the present study, the results of treated groups (BBR and BBR-loaded micelle) showed a significant decrease in TNF- $\alpha$, IL-1ß, MDA levels in comparison with the stroke group. This result confirmed by Zhang et al. that reported BBR could be suppressed the activation of proinflammatory cytokines (TNF- $\alpha$, IL1ß) after ischemic stroke [9]. This result indicates that the anti-inflammatory properties of berberine and an ameliorate effect against stress oxidative situation that happens due to the BCCAO model. Moreover, this property was further observed in the nano formulation.

Generally, we designed a useful nano formulation, prepared with BBR as drug and micelle as the carrier, BBRloaded micelle, by thin-film hydration method for reduction of inflammatory effect in cerebral ischemia in the rat. The results of the study suggest that prepared nano formulation possesses improved anti-inflammatory effects more compared to the usual formulation. This effect could be due to the increased aqua solubility in BBR-loaded micelle compared to BBR, resulting in its increased efficiency. Thus, BBR-loaded micelle formulation could be developed as a promising preventive agent on cerebral ischemia.

\section{Supplementary Information}

The online version contains supplementary material available at https://doi. org/10.1186/s40360-021-00525-7.

\section{Additional file 1. \\ Additional file 2. \\ Additional file 3 . \\ Additional file 4.}

\section{Acknowledgments}

The authors thank the Department of Pharmacology, Faculty of Medicine, Tehran University of Medical sciences, Tehran, Iran. (Grant No: 98023042949) Nanotechnology research center, Pharmaceutical technology institute, Mashhad University of Medical Sciences, Mashhad, Iran.

\section{The ARRIVE guidelines}

The study was carried out in compliance with ARRIVE guidelines 2.0.

\section{Authors' contributions}

RA and SEM contributed equally to the study conception, design, and preparation of the manuscript. HY, NM, and MRJ contributed to performing the experiments. HY, SEM, and MR, supervised the study and contributed to the critical revision of the manuscript. All the authors read and approved the final manuscript.

\section{Funding}

This study was financially supported by the Department of Pharmacology, Faculty of Medicine, Tehran University of Medical Sciences, Tehran, Iran. (Grant No: 98023042949).

Availability of data and materials

All the necessary data is included in the article. Further data will be shared by request.

\section{Declarations}

Ethics approval and consent to participate

All the experiments were in accordance with the guidelines of Tehran University of Medical Sciences and approved by the Animal Ethics Committee (IR.TUMS.MEDICINE.REC.1398.593).

Consent for publication

Not applicable.

\section{Competing interests}

The authors declare that they have no competing interests.

\section{Author details}

${ }^{1}$ Department of Medical Nanotechnology, Faculty of Advanced Sciences and Technology, Tehran Medical Sciences, Islamic Azad University, Tehran, Iran. ${ }^{2}$ Department of Pharmacology, School of Medicine, Tehran University of Medical Sciences, Tehran, Iran. ${ }^{3}$ Department of Medical Nanotechnology, School of Advanced Technologies in Medicine, Tehran University of Medical Sciences, Tehran, Iran. ${ }^{4}$ Department of Pharmaceutical Nanotechnology, Mashhad University of Medical Sciences, Mashhad, Iran.

Received: 8 December 2020 Accepted: 21 September 2021

Published online: 03 October 2021

\section{References}

1. Béjot $Y$, Daubail B, Giroud M. Epidemiology of stroke and transient ischemic attacks: current knowledge and perspectives. Rev Neurol (Paris). 2016;172(1): 59-68. https://doi.org/10.1016/j.neurol.2015.07.013.

2. Lee RHC, Lee MHH, Wu CYC, Couto E Silva A, Possoit HE, Hsieh TH, et al. Cerebral ischemia and neuroregeneration. Neural Regen Res. 2018;13(3): 373-85. https://doi.org/10.4103/1673-5374.228711.

3. Siniscalchi A, Gallelli L, Malferrari G, Pirritano D, Serra R, Santangelo E, et al. Cerebral stroke injury: the role of cytokines and brain inflammation. J Basic Clin Physiol Pharmacol. 2014;25(2):131-7. https://doi.org/10.1515/jbcpp-2 013-0121.

4. Cheng C-Y, Lee Y-C. Anti-inflammatory effects of traditional Chinese medicines against ischemic injury in in vivo models of cerebral ischemia. Evid Based Complement Alternat Med. 2016;2016:5739434-16. https://doi. org/10.1155/2016/5739434.

5. Lambertsen $\mathrm{KL}$, Biber $\mathrm{K}$, Finsen B. Inflammatory cytokines in experimental and human stroke. J Cereb Blood Flow Metab. 2012;32(9):1677-98. https:// doi.org/10.1038/jcbfm.2012.88.

6. Wang Q, Qi J, Hu R, Chen Y, Kijlstra A, Yang P. Effect of berberine on proinflammatory cytokine production by ARPE-19 cells following stimulation with tumor necrosis factor-a. Invest Ophthalmol Vis Sci. 2012;53(4):2395402. https://doi.org/10.1167/iovs.11-8982.

7. Srivastava S, Srivastava M, Misra A, Pandey G, Rawat A. A review on biological and chemical diversity in Berberis (Berberidaceae). EXCLI J. 2015; 14:247-67. https://doi.org/10.17179/excli2014-399.

8. Kim M, Kim TW, Kim CJ, Shin MS, Hong M, Park HS, et al. Berberine ameliorates brain inflammation in Poloxamer 407-induced Hyperlipidemic rats. Int Neurourol J. 2019;23(Suppl 2):S102-10. https://doi.org/10.5213/inj.1 938216.108

9. Zhang Q, et al. Treatment effects of ischemic stroke by Berberine, Baicalin, and Jasminoidin from Huang-Lian-Jie-Du-decoction (HLJDD) explored by an integrated metabolomics approach. Oxidative Med Cell Longev. 2017;2017: 9848594.

10. Yuan NN, Cai CZ, Wu MY, Su HX, Li M, Lu JH. Neuroprotective effects of berberine in animal models of Alzheimer's disease: a systematic review of pre-clinical studies. BMC Complement Altern Med. 2019;19(1):109. https:// doi.org/10.1186/s12906-019-2510-z. 
11. Tong $L$, Xie C, Wei $Y$, Qu Y, Liang $H$, Zhang $Y$, et al. Antitumor effects of Berberine on gliomas via inactivation of Caspase-1-mediated IL-1 $\beta$ and IL-18 release. Front Oncol. 2019;9:364. https://doi.org/10.3389/fonc.2019.00364.

12. Tan J, Wang J, Yang C, Zhu C, Guo G, Tang J, et al. Antimicrobial characteristics of Berberine against prosthetic joint infection-related Staphylococcus aureus of different multi-locus sequence types. BMC Complement Altern Med. 2019; 19(1):218. https://doi.org/10.1186/s12906-019-2558-9.

13. Rajasekhar K, et al. Antioxidant Berberine-Derivative Inhibits Multifaceted Amyloid Toxicity. iscience. 2020;23(4):101005.

14. Botwina P, Owczarek K, Rajfur Z, Ochman M, Urlik M, Nowakowska M, et al, Berberine hampers influenza a replication through inhibition of MAPK/ERK pathway. Viruses. 2020;12(3):344. https://doi.org/10.3390/v12030344.

15. Wang $C Y$, Bai $X Y$, Wang $C H$. Traditional Chinese medicine: a treasured natural resource of anticancer drug research and development. Am J Chin Med. 2014;42(3):543-59. https://doi.org/10.1142/S0192415X14500359.

16. Chen W, Miao YQ, Fan DJ, Yang SS, Lin X, Meng LK, et al. Bioavailability study of Berberine and the enhancing effects of TPGS on intestinal absorption in rats. AAPS PharmSciTech. 2011;12(2):705-11. https://doi.org/1 0.1208/s12249-011-9632-z.

17. Zuo F, Nakamura N, Akao T, Hattori M. Pharmacokinetics of berberine and its main metabolites in conventional and pseudo germ-free rats determined by liquid chromatography/ion trap mass spectrometry. Drug Metab Dispos. 2006;34(12):2064-72. https://doi.org/10.1124/dmd.106.011361.

18. Feng R, Zhao ZX, Ma SR, Guo F, Wang Y, Jiang JD. Gut microbiota-regulated pharmacokinetics of Berberine and active metabolites in beagle dogs after Oral administration. Front Pharmacol. 2018;9:214. https://doi.org/10.3389/ fphar.2018.00214.

19. Tsai $\mathrm{P}, \mathrm{Tsai} \mathrm{TH}$. Simultaneous determination of berberine in rat blood, liver and bile using microdialysis coupled to high-performance liquid chromatography. J Chromatogr A. 2002;961(1):125-30. https://doi.org/10.101 6/S0021-9673(02)00378-3.

20. Wang Z, Guo X, Liu Z, Cui M, Song F, Liu S. Studies on alkaloids binding to GC-rich human survivin promoter DNA using positive and negative ion electrospray ionization mass spectrometry. J Mass Spectrom. 2008:43(3):32735. https://doi.org/10.1002/jms.1320.

21. Wang $Z$, et al. Berberine-loaded Janus nanocarriers for magnetic fieldenhanced therapy against hepatocellular carcinoma. Chem Biol Drug Design. 2017:89(3):464-9.

22. Yu F, Ao M, Zheng X, Li N, Xia J, Li Y, et al. PEG-lipid-PLGA hybrid nanoparticles loaded with berberine-phospholipid complex to facilitate the oral delivery efficiency. Drug Delivery. 2017;24(1):825-33. https://doi.org/10.1 080/10717544.2017.1321062.

23. Wang T, Wang N, Song H, Xi X, Wang JA, Hao A, et al. Preparation of an anhydrous reverse micelle delivery system to enhance oral bioavailability and anti-diabetic efficacy of berberine. Eur J Pharma Sci. 2011:44(1-2):12735. https://doi.org/10.1016/j.ejps.2011.06.015

24. Chen $W$, Wei $S, Y u$ Y, Xue H, Yao F, Zhang M, et al. Pretreatment of rats with increased bioavailable berberine attenuates cerebral ischemiareperfusion injury via down regulation of adenosine-5'monophosphate kinase activity. Eur J Pharmacol. 2016;779:80-90. https://doi.org/10.1016/j. ejphar.2016.03.015

25. Fakhraei $\mathrm{N}$, et al. Berberine nanomicelles attenuate cirrhotic cardiomyopathy in rats: possible involvement of the NO-cGMP signaling. Nanomed J. 2020; 7(4):299-307.

26. Luo JW, Zhang ZR, Gong T, Fu Y. One-step self-assembled nanomicelles for improving the oral bioavailability of nimodipine. Int J Nanomedicine. 2016; 11:1051-65. https://doi.org/10.2147/IJN.S97834

27. Parikh A, Kathawala K, Song Y, Zhou XF, Garg S. Curcumin-loaded selfnanomicellizing solid dispersion system: part I: development, optimization, characterization, and oral bioavailability. Drug Deliv Transl Res. 2018;8(5): 1389-405. https://doi.org/10.1007/s13346-018-0543-3.

28. Jin Q, Cai Y, Li S, Liu H, Zhou X, Lu C, et al. Edaravone-encapsulated agonistic micelles rescue ischemic brain tissue by tuning blood-brain barrier permeability. Theranostics. 2017;7(4):884-98. https://doi.org/10.7150/thno.18219.

29. Patra JK, Das G, Fraceto LF, Campos EVR, Rodriguez-Torres MP, Acosta-Torres LS, et al. Nano based drug delivery systems: recent developments and future prospects. J Nanobiotechnol. 2018;16(1):71. https://doi.org/10.1186/ s12951-018-0392-8.

30. Gaucher G, Satturwar P, Jones MC, Furtos A, Leroux JC. Polymeric micelles for oral drug delivery. Eur J Pharm Biopharm. 2010;76(2):147-58. https://doi. org/10.1016/j.ejpb.2010.06.007.
31. Salimi A, Sharif B, Esfahani G. Polymeric micelle as a new carrier in Oral drug delivery systems. Asian J Pharma. 2017;11:S704.

32. Ai X, Zhong L, Niu H, He Z. Thin-film hydration preparation method and stability test of DOX-loaded disulfide-linked polyethylene glycol 5000-lysinedi-tocopherol succinate nanomicelles. Asian J Pharma Sci. 2014;9(5):244-50. https://doi.org/10.1016/j.ajps.2014.06.006.

33. Nik ME, Malaekeh-Nikouei B, Amin M, Hatamipour M, Teymouri M, Sadeghnia HR, et al. Liposomal formulation of Galbanic acid improved therapeutic efficacy of pegylated liposomal doxorubicin in mouse colon carcinoma. Sci Rep. 2019;9(1):9527. https://doi.org/10.1038/s41598-019-4 5974-7.

34. Hatamipour M, Sahebkar A, Alavizadeh SH, Dorri M, Jaafari MR. Novel nanomicelle formulation to enhance bioavailability and stability of curcuminoids. Iran J Basic Med Sci. 2019;22(3):282-9. https://doi.org/10.2203 8/ijbms.2019.32873.7852.

35. Turner PV, Brabb T, Pekow C, Vasbinder MA. Administration of substances to laboratory animals: routes of administration and factors to consider. J Am Assoc Lab Anim Sci. 2011;50(5):600-13.

36. Partoazar A, Nasoohi S, Rezayat SM, Gilani K, Mehr SE, Amani A, et al. Nanoliposome containing cyclosporine a reduced neuroinflammation responses and improved neurological activities in cerebral ischemia/ reperfusion in rat. Fundam Clin Pharmacol. 2017;31(2):185-93. https://doi. org/10.1111/fcp.12244.

37. Farbiszewski R, Bielawski K, Bielawska A, Sobaniec W. Spermine protects in vivo the antioxidant enzymes in transiently hypoperfused rat brain. Acta Neurobiol Exp (Wars). 1995;55(4):253-8.

38. Chen YF, Wang YW, Huang WS, Lee MM, Wood WG, Leung YM, et al. TransCinnamaldehyde, an essential oil in cinnamon powder, ameliorates cerebral ischemia-induced brain injury via inhibition of Neuroinflammation through attenuation of iNOS, COX-2 expression and NFK-B signaling pathway. NeuroMolecular Med. 2016;18(3):322-33. https://doi.org/10.1007/s12017-0168395-9.

39. Nayak VS, Kumar N, D'Souza AS, Nayak SS, Cheruku SP, Pai KSR. The effects of Mucuna pruriens extract on histopathological and biochemical features in the rat model of ischemia. Neuroreport. 2017;28(18):1195-201. https://doi. org/10.1097/WNR.0000000000000888.

40. Maleki SN, Aboutaleb N, Souri F. Berberine confers neuroprotection in coping with focal cerebral ischemia by targeting inflammatory cytokines. J Chem Neuroanat. 2018;87:54-9. https://doi.org/10.1016/j.jchemneu.2017.04. 008.

41. Singh DP, Chopra K. Verapamil augments the neuroprotectant action of berberine in rat model of transient global cerebral ischemia. Eur J Pharmacol. 2013;720(1):98-106. https://doi.org/10.1016/j.ejphar.2013.10.043.

42. Yousefi-Manesh H, Rashidian A, Hemmati S, Shirooie S, Sadeghi MA, Zarei N, et al. Therapeutic effects of modafinil in ischemic stroke; possible role of NFKB downregulation. Immunopharmacol Immunotoxicol. 2019;41(5):558-64. https://doi.org/10.1080/08923973.2019.1669045.

43. Simões SM, Fiqueiras AR, Veiga F, Concheiro A, Alvarez-Lorenzo C. Polymeric micelles for oral drug administration enabling locoregional and systemic treatments. Expert Opin Drug Deliv. 2015;12(2):297-318. https://doi.org/10.1 517/17425247.2015.960841

44. Li C, Li X. Development and validation of a method for determination of encapsulation efficiency of CPT-11/DSPE-mPEG2000 nanoparticles. Med Chem. 2016;6(05):1-4. https://doi.org/10.4172/2161-0444.1000368.

45. Wu L, Xiong X, Wu X, Ye Y, Jian Z, Zhi Z, et al. Targeting oxidative stress and inflammation to prevent ischemia-reperfusion injury. Front Mol Neurosci. 2020;13:28. https://doi.org/10.3389/fnmol.2020.00028.

\section{Publisher's Note}

Springer Nature remains neutral with regard to jurisdictional claims in published maps and institutional affiliations. 